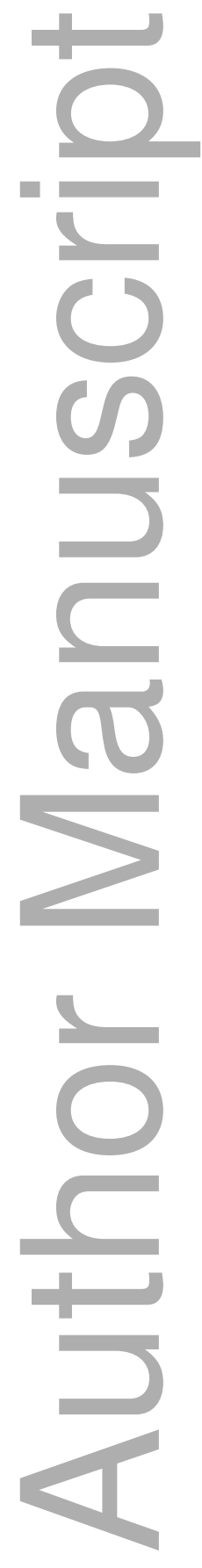

This is the author manuscript accepted for publication and has undergone full peer review but has not been through the copyediting, typesetting, pagination and proofreading process, which may lead to differences between this version and the Version of Record. Please cite this article as doi: $\underline{10.1111 / 1471-0528.16157}$

This article is protected by copyright. All rights reserved 
DR. JAMES MICHAEL NIALL DUFFY (Orcid ID : 0000-0001-6127-9859)

BEN WJ MOL (Orcid ID : 0000-0001-8337-550X)

DR. RUI WANG (AUSTRALIA) (Orcid ID : 0000-0002-6622-8134)

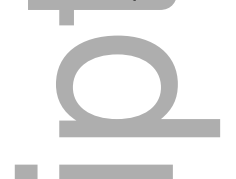

Article type : Main research article

\section{A core outcome set for future endometriosis research: an international consensus development study}

James M. N. Duffy ${ }^{1,2}{ }^{*}$, Martin Hirsch ${ }^{1,3}{ }^{*}$, Melissa Vercoe ${ }^{4}$, Jason Abbott ${ }^{5}$, Claire Barker ${ }^{6}$, Barbara Collura 7, Robyn Drake ${ }^{8}$, Johannes L.H. Evers ${ }^{9}$, Martha Hickey ${ }^{10}$, Andrew W. Horne ${ }^{11}$, Louise Hull ${ }^{12}$, Sharada Kolekar ${ }^{8}$, Sarah Lensen ${ }^{4}$, Neil P. Johnson ${ }^{12,13}$, Vishakha Mahajan ${ }^{8}$, Ben Mol ${ }^{14}$, Anne-Sophie Otter ${ }^{15}$, Lucian Puscasiu ${ }^{16}$, Magdalena Bofill Rodriguez ${ }^{4}$, Luk Rombauts ${ }^{14}$, Andy Vail ${ }^{17}$, Rui Wang ${ }^{12}$, Cindy M. Farquhar ${ }^{4}$ and endo:outcomes An International Collaboration Harmonising Outcomes and Outcome Measures for Endometriosis Research (contributors listed at the end of the manuscript)

${ }^{1}$ Institute for Women's Health, University College London, London, United Kingdom.

2 Barking, Havering and Redbridge University Hospitals NHS Trust, London, United Kingdom.

3 University College London Hospitals NHS Foundation Trust, London, United Kingdom.

${ }^{4}$ Cochrane Gynaecology and Fertility Group, University of Auckland, Auckland, New Zealand. ${ }^{5}$ School of Women's and Children's Health, University of New South Wales Sydney, Sydney, Australia.

${ }^{6}$ Radcliffe Women's Health Patient Participation Group, University of Oxford, Oxford, United Kingdom.

${ }^{7}$ Resolve: The National Infertility Association, Virginia, United States.

${ }^{8}$ endo:outcomes Patient and Public Participation Group, University of Auckland, Auckland, New Zealand.

This article is protected by copyright. All rights reserved 
${ }^{9}$ Centre for Reproductive Medicine and Biology, University Medical Centre Maastricht, Maastricht, The Netherlands.

10 Department of Obstetrics and Gynaecology, University of Melbourne, Victoria, Australia.

${ }^{11}$ MRC Centre for Reproductive Health, University of Edinburgh, Edinburgh, United Kingdom.

12 Robinson Research Institute, University of Adelaide, Adelaide, South Australia, Australia.

13 World Endometriosis Society, Vancouver, Canada.

14 Department of Obstetrics and Gynaecology, School of Medicine, Monash University, Melbourne, Australia.

15 Osakidetza, OSı Bilbao, Basurto, Spain.

16 University of Medicine, Pharmacy, Science, and Technology, Targu Mures, Romania.

${ }^{17}$ Centre for Biostatistics, University of Manchester, Manchester Academic Health Science Centre, Manchester, United Kingdom.

* equal contribution

\section{Correspondence to}

Dr James M. N. Duffy DPhil MRes PG HCL MBChB BSc (Hons)

Institute for Women's Health, University College London

London WC1E 6BT

United Kingdom

+447949066806

james.duffy3@nhs.net

@jamesmnduffy

\section{Running title}

A core outcome set for endometriosis

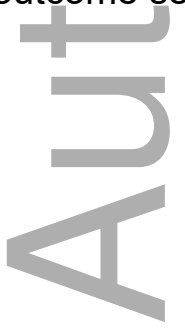

This article is protected by copyright. All rights reserved 


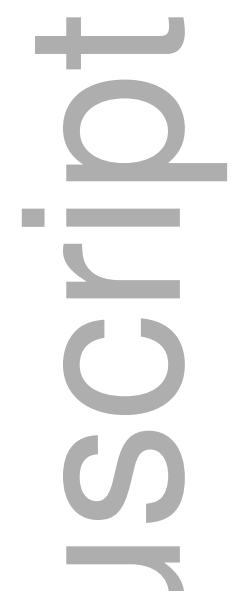

Objective To develop a core outcome set for endometriosis.

Design Consensus development study.

Setting International.

Population One hundred and sixteen healthcare professionals, 31 researchers, and 206 patient representatives.

Methods Modified Delphi method and modified Nominal Group Technique.

Results The final core outcome set includes three core outcomes for trials evaluating potential treatments for pain and other symptoms associated with endometriosis: overall pain, improvement in the most troublesome symptom, and quality of life. In addition, eight core outcomes for trials evaluating potential treatments for infertility associated with endometriosis were identified: viable intrauterine pregnancy confirmed by ultrasound, pregnancy loss including ectopic pregnancy, miscarriage, stillbirth, and termination of pregnancy, live birth, time to pregnancy leading to live birth, gestational age at delivery, birth weight, neonatal mortality, and major congenital abnormalities. Two core outcomes applicable to all trials were also identified: adverse events and patient satisfaction with treatment. 
Conclusions Using robust consensus science methods, healthcare professionals, researchers, and women with endometriosis have developed a core outcome set to standardise outcome selection, collection, and reporting across future randomised controlled trials and systematic reviews evaluating potential treatments for endometriosis.

Funding Royal Society of New Zealand, New Zealand and Royal College of Obstetricians and Gynaecologists, United Kingdom.

Keywords Consensus development study, core outcome set, endometriosis, modified Delphi method, and modified Nominal Group Technique.

Tweetable abstract @coreoutcomes for future \#endometriosis research have been developed @jamesmnduffy

\section{Introduction}

Endometriosis research should inform clinical practice and in doing so improve the treatment outcomes of women with endometriosis. For this to be possible, randomised controlled trials should select, collect, and report outcomes which reflect the realities of everyday clinical practice and are relevant to women with endometriosis. ${ }^{1,2}$ Unfortunately, many endometriosis trials fall short in this regard. For example, when considering randomised trials evaluating potential surgical treatments for infertility associated with endometriosis, only a minority reported live birth $(5 / 32 ; 15 \%)$, pregnancy loss $(7 / 32 ; 22 \%)$, and adverse events $(9 / 32 ; 28 \%) .{ }^{3}$ In addition, substantial variation exists in the measurement instruments used to collect individual outcomes. For example, dysmenorrhea has been measured using ten different measurement instruments including visual analogue scales, ranked ordinal scales, and symptom questionnaires. ${ }^{3}$ Combining different measurement instruments within a meta-analysis is challenging and weakens the reliability of the final summary estimate. ${ }^{4}$ Such variation can result in individual 
researchers reporting outcomes on the basis of statistical significance, which can skew an entire evidence base to overestimate the benefits of treatments and underestimate the harms. ${ }^{1}$

These challenges can be addressed by developing, disseminating, and implementing a minimum data set, termed a core outcome set, to standardise the selection, measurement, and reporting of outcomes across randomised controlled trials and systematic reviews. They are developed in three stages. The first stage is to develop a long list of potential core outcomes by undertaking a systematic review of published randomised controlled trials. The next stage is to reduce the long list of potential core outcomes to a core outcome set using formal consensus methods. The final stage is to determine how individual core outcomes should be defined and measured.

Motivated by the desire to improve endometriosis research, an international collaboration embedded with the Cochrane Gynaecology and Fertility Group, has brought healthcare professionals, researchers, and women with endometriosis together to develop a core outcome set for future endometriosis research.

\section{Methods}

The study was prospectively registered with the Core Outcome Measures in Effectiveness Trials (COMET) initiative, registration number 1023. An international steering group, including healthcare professionals, researchers, and patient representatives, was established. A protocol describing the study's methods has been published. ${ }^{5}$

The core outcome set was developed in a three-stage process using methods advocated by the COMET initiative. ${ }^{6}$ Potential core outcomes were identified through a systematic review of 
endometriosis trials which has previously been published. ${ }^{3}$ The long list of potential core outcomes was entered into a modified Delphi method which facilitated individual stakeholder group convergence towards consensus outcomes. These outcomes were entered into a face to face consensus development meeting. Using a modified Nominal Group Technique, consensus outcomes were further prioritised to identify the final core outcome set for endometriosis.

We performed a systematic review of published trials evaluating the potential treatments for endometriosis and extracted the reported outcomes. ${ }^{3}$ A comprehensive inventory of outcomes was developed in consultation with the study's steering group. Who were encouraged to remove similar outcomes described using different terminology (for example, deep vein thrombosis, lower limb thrombosis, pulmonary embolism, embolism, venous thromboembolism), non-patient-centered outcomes (for example, inflammatory markers), and outcomes specific to individual experimental interventions (for example, side effects associated with hormone replacement therapy). Lay definitions were developed for individual outcomes in consultation with the study's public and patient research partners and with reference to established medical terminology directories developed by the National Institute for Health Research, the Royal College of Obstetricians and Gynaecologists, the State Government of Victoria, and Wiley. ${ }^{7-10}$ These outcomes and lay definitions were entered into the modified Delphi method which was delivered through sequential online surveys using Delphi survey software (Delphi Manager, University of Liverpool, Liverpool, United Kingdom).

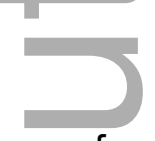

Healthcare professionals, researchers, and women with endometriosis were invited to participate. Recruitment was supported by the British Society of Gynaecological Endoscopy, Cochrane Gynaecology and Fertility group, Endometriosis Clinical Study Group, World Endometriosis Society, and an active social media campaign. The Delphi method does not depend on statistical power, therefore, group error should reduce as the number of participants

This article is protected by copyright. All rights reserved 
increases. ${ }^{11}$ Between ten and 15 participants have been demonstrated to yield sufficient results and assure validity. ${ }^{11-13}$ We aimed to recruit at least 18 participants for each stakeholder group, anticipating an overall attrition rate of $20 \%$ between Delphi survey rounds.

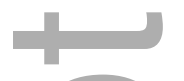

In round one, participants scored individual outcomes on a nine-point Likert scale anchored between one (labelled 'not important') and nine (labelled 'critical'). ${ }^{14}$ This scale was devised by the Grading of Recommendations Assessment, Development and Evaluation (GRADE) working group to facilitate the ranking of outcomes according to their importance and has been adopted widely by core outcome set developers. Participants were able to select an 'unable to score' category if they did not have enough expertise or experience to score an individual outcome. At the end of the survey, participants were able to suggest additional outcomes. After the round one survey had closed, the scores for each outcome were aggregated across individual stakeholder groups. The percentage of participants scoring each outcome at every possible response from one to nine was calculated by the Delphi survey software and tabulated for individual stakeholder groups (healthcare professionals, researchers, and women with endometriosis). Additional outcomes were considered by the steering group and outcomes which had not been present in round one were entered into round two.

In round two, participants received their own scores and individual stakeholder group feedback for each round one outcome. Participants were asked to reflect on their own scores and on the scores of other participants, before rescoring each outcome. Before completing the survey, participants were able to score additional outcomes suggested by participants in the round one Delphi survey. The $70 \% / 15 \%$ consensus definition advocated by the COMET initiative was applied to the round two Delphi survey results. ${ }^{6} \mathrm{~A}$ consensus outcome was identified when over $70 \%$ of participants in each stakeholder group scored the outcome 'critical for decision making' 
(score seven to nine) and less than $15 \%$ of participants in each stakeholder group scored the outcome 'of limited importance for decision making' (score one to three).

Following the Delphi survey, a face-to-face consensus development meeting was arranged to discuss the survey results. The consensus development meeting used a modified Nominal Group Technique technique to further prioritise consensus outcomes. Healthcare professionals, researchers, and women with endometriosis were invited to participate. The modified nominal group technique does not depend on statistical power. We aimed to recruit between ten and 15 participants, as this number has yielded sufficient results and assured validity in other settings. ${ }^{11,15}$

The modified Nominal Group Technique was delivered through a half day consensus development conference. At the start of the meeting, the results of the Delphi survey were reviewed, and all consensus outcomes were entered into the process. Participants added two further outcomes which had not been scored in the Delphi survey: termination of pregnancy and improvement in the most troublesome symptom. Each participant was asked to contribute their opinions on the suitability of individual outcomes forming a component of the final core outcome set. Participants were encouraged to reformulate outcomes to improve clarity or comprehension and where appropriate group outcomes in an outcome domain. For example, damage to bowel, damage to the nervous system, and damage to renal tract, were grouped into an outcome domain labelled adverse events. This would enable a degree of flexibility to tailor adverse event reporting in future endometriosis trials to the experimental intervention being evaluated.

Following the initial discussion, outcomes were divided into three initial categories: (1) outcomes to be considered for inclusion in the final core outcome set; (2) outcomes where no consensus existed; and (3) outcomes which should not be considered for inclusion in the final core outcome set. Participants were invited to discuss the ordering of the outcomes within each

This article is protected by copyright. All rights reserved 
category. The discussion focused upon ranking the outcomes being considered for inclusion in the final core outcome set and the outcomes where no consensus existed. During the discussion, the outcomes could be moved between the categories. Following the discussion, the final core outcome set for endometriosis was agreed.

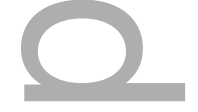

This study was funded by the Catalyst Fund, Royal Society of New Zealand, New Zealand and Endometriosis Millennium Fund, Royal College of Obstetricians and Gynaecologists, United Kingdom. The funders have no role in the design and conduct of the study, the collection, management, analysis, or interpretation of data, or manuscript preparation.

\section{Results}

When considering the Delphi survey, round one was completed by 354 participants representing 25 countries (Table 1). Round two was completed by 238 of the original participants (67\%).

Fifty-five outcomes were entered into the round one Delphi survey (figure 1). In response to the outcomes suggested by participants, the steering group included 34 new outcomes to round two (Appendix S1). Therefore, 89 outcomes were entered into round two. Following round two, 18 outcomes reached consensus and were entered into the consensus development conference.

Twenty-four participants, representing seven countries, participated in the consensus development meeting. Eighteen consensus outcomes were entered into the modified Nominal Group Technique. Participants entered an additional ten no consensus outcomes into the process, including physical functioning, spontaneous conception following medical or surgical treatment of endometriosis, and cumulative live birth rate. Therefore, 28 potential core 
outcomes were discussed in total. Participants prioritised thirteen outcomes for inclusion in the core outcome set for future endometriosis research (figure 1).

\section{Discussion

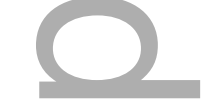

\section{Main findings}

Using robust consensus science methods, healthcare professionals, researchers, and women with endometriosis have developed a core outcome set to standardise outcome selection, collection, and reporting across future randomised controlled trials and systematic reviews evaluating potential treatments for endometriosis. The core outcome set is applicable to potential treatments for pain, infertility, and other symptoms associated with endometriosis.

\section{Strengths and limitations}

Our core outcome set development study met the methodological standards for core outcome set development recently published by the COMET initiative. ${ }^{16}$ All eleven standards were achieved across three broad domains including scope specification, stakeholder involvement, and consensus development process. When considering the Delphi survey, 354 participants, from 25 countries, engaged with the prioritisation of outcomes. The study included women with endometriosis as both steering group members and as participants. As steering group members, they provided valuable oversight, design, and development of the Delphi survey. As participants, they shared their views during the Delphi survey and contributed to the prioritisation of outcomes for inclusion within the final core outcome set during the consensus development meeting.

This article is protected by copyright. All rights reserved 
This consensus study is not without limitations. Consideration should be given to the representative of the study's participants. For example, when considering the Delphi survey, there was a higher response from participants who lived in Europe (225 participants; 63\%). We appreciate limitations in the representative of the sample could have impacted upon the outcomes prioritised. The best approaches to combining the views of different stakeholders have been rarely investigated in previous core outcome set development studies. Several studies have included a heterogeneous group of participants representing multiple stakeholder groups included in a single panel. ${ }^{17}$ When these data were combined, with no consideration of the separate stakeholder groups, the resulting set depended upon the relative proportions of the individual stakeholder groups participating. Further methodological research is required to explore panel size, panel composition, and consensus definition.

The Delphi survey's attrition rate was $33 \%$, which is comparable to other core outcome development studies. ${ }^{17}$ It may be possible to reduce attrition by reducing the number of participants, removing outcomes which reached consensus in subsequent survey rounds, or reducing the number of survey rounds. However, attrition needed to be balanced with the requirement to encourage a diverse range of stakeholders to participate, entering a comprehensive long list of potential core outcomes into the Delphi survey, and for participants to be able to reflect on and rescore individual outcomes in relation to each other.

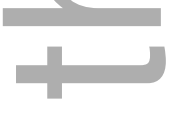

The a priori consensus definition used in this study could be perceived as another potential limitation. The modified Delphi method used the $70 \% / 15 \%$ consensus definition which is subjective and not based upon research evidence or statistical evaluation. ${ }^{6}$ Further methodological research is required to develop an appropriate consensus definition. It is likely this definition would need to be dynamic with specific conditions or combination criteria which could consider the unique scoring distribution of the Delphi survey participants.

This article is protected by copyright. All rights reserved 


\section{Interpretation}

A recent research prioritisation exercise has highlighted the most pressing research questions as perceived by healthcare professionals and women with endometriosis. ${ }^{18}$ Many of these research questions will require the evaluation of potential treatments for endometriosis within a randomised controlled trial setting. Complex issues, including a failure to take into account the perspectives of women with endometriosis when designing trials, variations in outcome measures, and outcome reporting bias, could undermine the translation of future endometriosis research into clinical practice. The core outcome set for endometriosis should standardise outcome collection and reporting across future endometriosis trials and will help to ensure future research ultimately improve care women with endometriosis receive.

Live birth is considered the most appropriate primary outcome for trials evaluating potential treatments for infertility. Following up trial participants for over nine months could have implications for the resources individual trials require. The development of a core outcome set for endometriosis should provide additional leverage for researchers to request sufficient funding to enable the collection of core outcomes, including live birth. The core outcome set presented an opportunity standardise the collection of outcomes, for example, pregnancy can be confirmed by the urinary beta human chorionic gonadotropin (HCG), serum beta HCG, and ultrasound. When presented with different outcomes prioritised by the Delphi survey the modified Nominal Group Technique enabled participates to rank different outcomes and make a recommendation, in this case, viable intrauterine pregnancy confirmed by ultrasound. Several core outcomes, including gestational age at delivery, birth weight, and neonatal mortality, were included to provide important safety signals for future randomised trials.

This article is protected by copyright. All rights reserved 
It is considered good practice for researchers developing clinical trial protocols to use the Standard Protocol Items: Recommendations for Interventional Trials (SPIRIT) statement. ${ }^{19}$ This statement outlines the scientific, ethical, and administrative elements that should be addressed and specifically recommends the use of core outcome sets where they exist.

The Core Outcomes in Women's and Newborn Health (CROWN) initiative aims to tackle poor outcome selection, collection, and reporting within our speciality. ${ }^{17}$ Participating journals will initially strongly encourage, and over the longer term require, researchers to report the core outcome set for endometriosis within randomised trial reports and offer conclusions based on these outcomes. Where core outcomes have not been collected, the researchers will be asked to report this deficiency and its implications for their findings. ${ }^{20}$

Objectively demonstrating the uptake of the core outcome set for endometriosis will be important in quantifying its contribution in tackling research waste by reducing the use of poorly selected, collected, and reported outcomes. Assessing the uptake of the core outcome set for endometriosis will be undertaken by examining registry records, published protocols, randomised controlled trials, and systematic reviews, and undertaking a citation analysis. In addition, further research is planned to examine and understand the reasons why researchers do, and do not, implement the core outcome set for endometriosis.

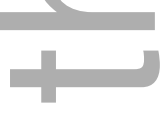

This study is complimentary to other initiatives the endometriosis research community has previously engaged with standardising important aspects of research design. The Art and Science of Endometriosis meeting, convened by the National Institutes of Health, has published recommendations regarding the standardisation of research design in several areas including entry criteria and outcome measures for pain symptoms. The World Endometriosis Research Foundation Endometriosis Phenome and Biobanking Harmonisation Project has published tools

This article is protected by copyright. All rights reserved 
for the standardisation of research design in several areas including clinical, covariate and surgical phenotype recording and specimen collection, processing and storage.

The work will continue, involving global participants from a range of stakeholder groups including healthcare professionals, researchers, industry representatives and patient representatives, reflecting the enthusiasm of our specialty to work together to improve research design, clinical research conduct, and clinical care.

\section{Conclusion}

This study has developed a core outcome set which should be implemented across future randomised trials and systematic reviews evaluating potential treatments for endometriosis to standardise outcome selection, collection, and reporting. Future research is required to associate core outcomes with high quality definitions and measurement instruments. The core outcome set for endometriosis will be reviewed every three years or in response to a paradigm shift in diagnosis, treatment, or understanding.

\section{endo:outcomes An International Collaboration Harmonising Outcomes and Outcome Measures for Endometriosis Research}

Professor Jane P Daniels, Nottingham Clinical Trials Unit, University of Nottingham, United Kingdom; Miss C M S, New Zealand; Dr Arianne C Lim, Maastrict University Medical Center, Netherlands; Mrs Katie Edmonds, HealEndo.com, United States of America; Mrs Claire E MacLean, Portugal; Dr James M N Duffy, Balliol College, University of Oxford, United Kingdom; Ms Amy C Appleton, Australia; Ms Sarah Skelton, United Kingdom; Mr Dominic L Byrne, Royal Cornwall Hospital, United Kingdom; Miss Rebecca K White, United Kingdom; Dr Margarida Sardo, United Kingdom; Ms Rebecca C Fowles, United Kingdom; Professor Baris Ata, Koc University of Medicine, Department of Obstetrics and Gynecology, Turkey; Miss Sarah A

This article is protected by copyright. All rights reserved 
Richatd, United Kingdom; Mrs Casandra C Puig Ybanez, United Kingdom; Professor Matthew C Peterson, University of Utah, Division of Reproductive Endocrinology and Infertility, Department of Obstetrics and Gynecology, United States of America; Miss Jasmin L Elms, Australia; Mrs Ann Parks, United Kingdom; Mrs Bianca L F De Bie Rocks, Endometriose Stichting, Netherlands; Miss Jodie C Roe, Australia; Ms Ruby Doran, United Kingdom; Dr Marcello Ceccaroni, Sacred Heart Hospital, Department of gynaecology and obstetrics, Italy; Mrs Ceu A Ferreira, Portugal; Professor Sofia Dias, University of Bristol, United Kingdom; Mrs Tracey Pinnington, United Kingdom; Mrs Paula Laupa-Santiago, France; Miss Marie C Turner, United Kingdom; Miss Anneke M F Schreurs, Amsterdam UMC, Vrije Universiteit Amsterdam, Netherlands; Mrs Eleanor Baggot, Walsall Healthcare Trust, United Kingdom; Professor Razvan V Socolov, University of Medicine and Pharmacy Gr T Popa lasi, Department of Obstetrics and Gynecology, Romania; Mr Menem Yossry, City Hospital Sunderland, United Kingdom; Miss Tania M Hodges, Miss Euroasia International, Malaysia; Dr Marina W P Barbossa, Genesis Human Reproduction Centre, Brazil; Professor Lucian Puscasiu, University of Medicine, Pharmacy, Science and Technology, Târgu Mures, Department of Obstetrics and Gynecology, Romania; Ms Tracy L Lytwyn, United States of America; Mrs Sophia E L Egan-Reid, New Zealand; Mrs Susanna C L Devlin, United Kingdom; Ms Kira S Crees, Australia; Ms Bethany C Baldwin, United States of America; Professor James R Scott, University of lowa, United States of America; Miss Amy K Gravolin, Australia; Miss Errin F Chapman, Canada; Dr Stephanie N Bartley, University of Western Australia, Faculty of Health and Medicine Sciences, Australia; Ms Alicia J Hamilton, Canada; Mrs Kirstie J Thorpe, New Zealand; Mrs Denise M Carmody, Australia; Miss Clare Joseph, United Kingdom; Dr Joanna Eyeson, United Kingdom; Miss Madeleine D Davis, Australia; Associate Professor Louise Hull, Robinson Research Institute, University of Adelaide, Australia; Ms Jo Henry, Ireland; Dr Mike Armour, NICM Heath Research Institute, Western Sydney University, Australia; Miss Presley Y F V Cummings, Canada; and Miss Lisa A Cook, United Kingdom.

This article is protected by copyright. All rights reserved 


\section{Acknowledgements}

We would like to thank the healthcare professionals, researchers, and patient representatives who participated in the Delphi survey and consensus development meetings.

\section{Conflicts of interest}

MLH has received travel grants from Merck-Serono and Geurbet; research funding from AbbVie, Merck, Origio and Myovant, and has been a consultant to Vifor Pharma.

AWH has received consultancy fees from AbbVie, Ferring, Nordic Pharma, and Roche Diagnostics. NPJ has received conference expenses from Bayer Pharma, Merck-Serono, and Merck and Co; research funding from AbbVie; and has been a consultant to Guerbet, Mvovant Sciences, and Vifor Pharma. LR has received research funding from Ferring Australia, MerckSerono, and MSD; and chairs the Ferring Australia advisory board. The remaining authors report no competing interests. Completed disclosure of interest forms are available to view online as supporting information.

\section{Author contributions}

Study concept and design: JMD, MH, MV, CB, SL, NPJ, BM, and CMF. Acquisition of data: JMD, MH, MV, SL, NPJ, BM, and CMF. Analysis and interpretation of data: JMD, MH, MV, JB, CB, BC, RD, JLE, MH, AWH, LH, SK, SL, NPJ, VM, BM, AO, LP, MBR, LR, AV, RW, and CMF. Drafting of the manuscript: JMD, MH, and CMF. Critical revision of the manuscript for important intellectual content: MV, JB, CB, BC, RD, JLE, MH, AWH, LH, SK, SL, NPJ, VM, BM, AO, LP, MBR, LR, AV, and RW. Obtaining funding: JMD, MH, and CMF. Study supervision: CMF.

\section{Sources of funding}

This article is protected by copyright. All rights reserved 
This study was funded by the Catalyst Fund, Royal Society of New Zealand, New Zealand and Endometriosis Millennium Fund, Royal College of Obstetricians and Gynaecologists, United Kingdom. The funders have no role in the design and conduct of the study, the collection, management, analysis, or interpretation of data, or manuscript preparation.

\section{Ethical approval}

Ethics approval was not required as the study was not a clinical trial, did not assess a device or expose a patient to ionising radiation, did not require collection or storage of any material / specimens / protected information, recruit patients / carers through the NHS, involve anyone with lack of capacity or prisoners, or xenotransplantation, and was not a social care project funded through the Department of Health.

\section{References}

1. Duffy JMN, Ziebland S, von Dadelszen P, McManus RJ. Tackling poorly selected, collected, and reported outcomes in obstetrics and gynecology research. American Journal of Obstetrics \& Gynecology. 2019;220(1):71.e1-.e4.

2. Johnson NP, Hummelshoj L, Consortium ftWESM, Abrao MS, Adamson GD, Allaire C, et al. Consensus on current management of endometriosis. Human Reproduction. 2013;28(6):1552-68.

3. Hirsch M, Duffy JMN, Kusznir JO, Davis CJ, Plana MN, Khan KS, et al. Variation in outcome reporting in endometriosis trials: a systematic review. American Journal of Obstetrics and Gynecology. 2016;214(4):452-64.

4. Duffy J, Bhattacharya S, Herman M, Mol B, Vail A, Wilkinson J, et al. Reducing research waste in benign gynaecology and fertility research. BJOG: An International Journal of Obstetrics \& Gynaecology. 2017;124(3):366-9.

5. Hirsch M, Duffy JMN, Barker C, Hummelshoj L, Johnson NP, Mol B, et al. Protocol for developing, disseminating and implementing a core outcome set for endometriosis. BMJ Open. 2016;6(12):e013998.

This article is protected by copyright. All rights reserved 
6. Williamson PR, Altman DG, Bagley H, Barnes KL, Blazeby JM, Brookes ST, et al. The COMET Handbook: Version 1.0. Trials. 2017;18(S3):280.

7. National Institute for Health Research. Jargon Buster. [cited; Available from: http://www.invo.org.uk/resource-centre/jargon-buster/ (Accessed 10 March 2015)

8. Royal College of Obstetricians and Gynaecologists. Medical terms explained. [cited; Available from: https://www.rcog.org.uk/en/patients/medical-terms/ (Accessed 10 December 2014)

9. State Government of Victoria. Medical terms and definitions during pregnancy and birth. [cited; Available from: https://www.betterhealth.vic.gov.au/health/servicesandsupport/medicalterms-and-definitions-during-pregnancy-and-birth (Accessed 12 November 2016)

10. Henderson B, Dorsey J. Medical Terminology for Dummies. Indianapolis, United States: Wiley Publishing, 2012.

11. Murphy M, Sanderson C, Black N, Askham J, Lamping D, Marteau T, et al. Consensus development methods, and their use in clinical guideline development. Health Technology Assessment. 1998;2(3):1-88.

12. Giannarou L, Zervas E. Using Delphi technique to build consensus in practice. International Journal of Business Science and Applied Management. 2014;9(2):65-82.

13. Skulmoski GJ, Hartman FT, Krahn J. The Delphi method for graduate research. Journal of Information Technology Education: Research. 2007;6:1-21.

14. Guyatt GH, Oxman AD, Kunz R, Atkins D, Brozek J, Vist G, et al. GRADE guidelines: 2. Framing the question and deciding on important outcomes. Journal of Clinical Epidemiology. 2011;64(4):395-400.

15. Gallagher M, Hares TIM, Spencer J, Bradshaw C, Webb IAN. The nominal group technique: A research tool for general practice? Family Practice. 1993;10(1):76-81.

16. Kirkham JJ, Davis K, Altman DG, Blazeby JM, Clarke M, Tunis S, et al. Core Outcome Set-STAndards for Development: The COS-STAD recommendations. PLOS Medicine. 2017;14(11):e1002447.

17. Duffy JMN, Rolph R, Gale C, Hirsch M, Khan KS, Ziebland S, et al. Core outcome sets in women's and newborn health: A systematic review. BJOG: An International Journal of Obstetrics and Gynaecology. 2017;124(10):1481-9.

18. Horne AW, Saunders PTK, Abokhrais IM, Hogg L. Top ten endometriosis research priorities in the UK and Ireland. The Lancet. 2017;389(10085):2191-2.

19. Chan A, Tetzlaff JM, Gøtzsche PC, Altman DG, Mann H, Berlin JA, et al. SPIRIT 2013 explanation and elaboration: guidance for protocols of clinical trials. BMJ. 2013;346:e7586.

This article is protected by copyright. All rights reserved 
20. Khan KS, Romero R. The CROWN Initiative: journal editors invite researchers to develop core outcomes in women's health. American Journal of Obstetrics \& Gynecology. 2014;211(6):575-6.
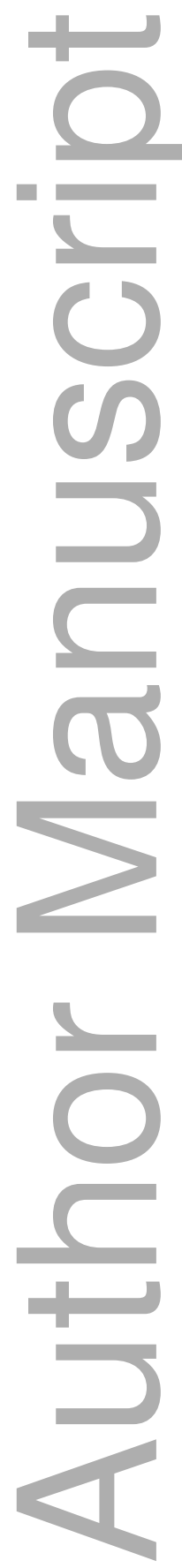
Figure 1 Flow of participants and outcomes

\section{Systematic review}

\section{Delphi survey round 1}

116 healthcare professionals

32 researchers

206 women with endometriosis

Delphi survey round 2

91 healthcare professionals 28 researchers

119 women with endometriosis
55 potential core outcomes

55 potential core outcomes

34 additional outcomes entered

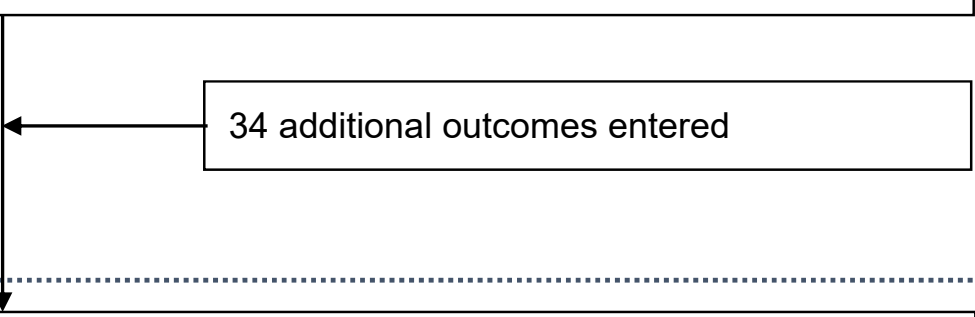

89 potential core outcomes scored

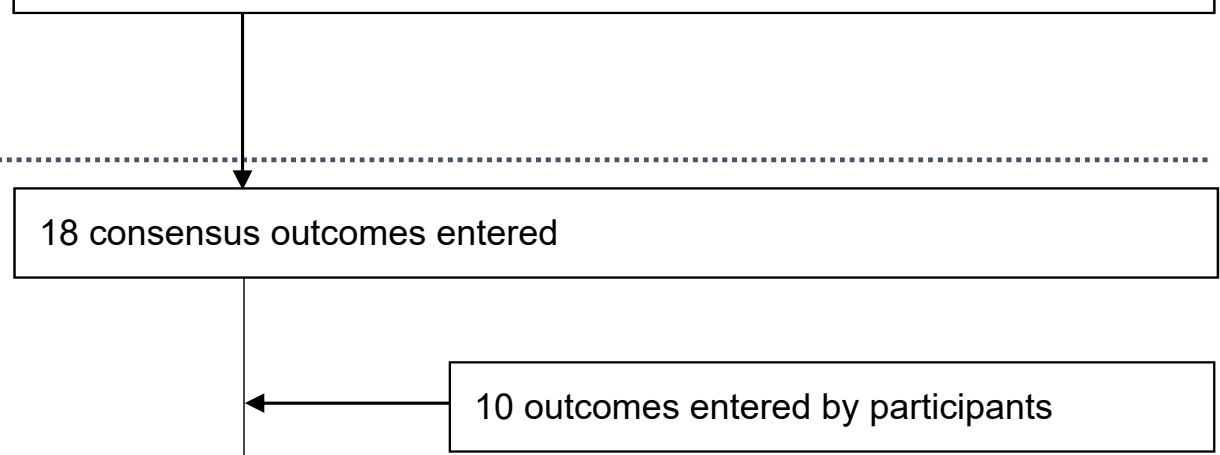

\section{Consensus meeting}

8 healthcare professionals

6 researchers

10 patients

\section{Final consensus}

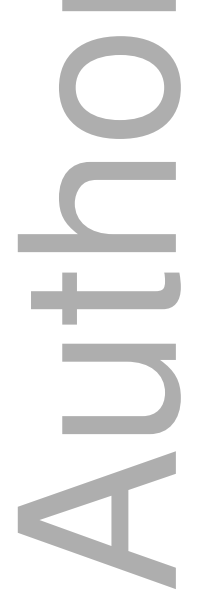

\section{Core outcome set for endometriosis}

- Overall pain

- Improvement in the most troublesome symptom

- Quality of life

- Viable intrauterine pregnancy confirmed by ultrasound

- Pregnancy loss including ectopic pregnancy, miscarriage, stillbirth, and termination of pregnancy

- Live birth

- Time to pregnancy leading to live birth

- Gestational age at delivery

- Birth weight

- Neonatal mortality

- Major congenital abnormalities

- Adverse events

- Patient satisfaction with treatment 
Table 1 Participant characteristics.

\begin{tabular}{|c|c|c|c|}
\hline \multirow{2}{*}{ Stakeholder group, $\mathbf{n}$} & \multicolumn{2}{|c|}{ Modified Delphi method } & \multirow{2}{*}{$\begin{array}{l}\text { Modified Nominal } \\
\text { Group technique }\end{array}$} \\
\hline & $\begin{array}{l}\text { Round } 1 \\
n=354\end{array}$ & $\begin{array}{l}\text { Round } 2 \\
n=238\end{array}$ & \\
\hline & & & \\
\hline Health professionals & 116 & 91 & 8 \\
\hline Gynaecologist with an interest in infertility & 23 & 22 & 2 \\
\hline Gynaecologist with an interest in pain & 26 & 21 & 3 \\
\hline Gynaecologist with an interest in pain and infertility & 51 & 48 & 2 \\
\hline Other & 16 & 14 & 1 \\
\hline Researchers & 32 & 28 & 6 \\
\hline Women with endometriosis & 206 & 119 & 10 \\
\hline \multicolumn{4}{|l|}{ Gender, n } \\
\hline Male & 79 & 61 & 11 \\
\hline Female & 273 & 176 & 13 \\
\hline Not stated & 2 & 1 & 0 \\
\hline Under 29 & 88 & 51 & 2 \\
\hline 30 to 39 & 113 & 79 & 9 \\
\hline 40 to 49 & 85 & 62 & 5 \\
\hline 50 to 59 & 51 & 36 & 5 \\
\hline Over 60 & 15 & 9 & 4 \\
\hline Prefer not to say & 2 & 1 & 3 \\
\hline \multicolumn{4}{|l|}{ Geographical location, n } \\
\hline Africa & 3 & 2 & 0 \\
\hline Asia & 5 & 4 & 0 \\
\hline Australia and New Zealand & 63 & 52 & 13 \\
\hline Europe & 225 & 132 & 9 \\
\hline North America & 51 & 44 & 2 \\
\hline Middle East & 2 & 1 & 0 \\
\hline South America & 3 & 2 & 0 \\
\hline Prefer not to say & 2 & 1 & 0 \\
\hline
\end{tabular}




\section{University Library}

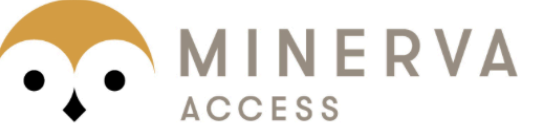

A gateway to Melbourne's research publications

Minerva Access is the Institutional Repository of The University of Melbourne

\section{Author/s:}

Duffy, JMN;Hirsch, M;Vercoe, M;Abbott, J;Barker, C;Collura, B;Drake, R;Evers, JLH;Hickey, M;Horne, AW;Hull, ML;Kolekar, S;Lensen, S;Johnson, NP;Mahajan, V;Mol, BW;Otter, AS;Puscasiu, L;Rodriguez, MB;Rombauts, L;Vail, A;Wang, R;Farquhar, CM

Title:

A core outcome set for future endometriosis research: an international consensus development study

\section{Date:}

2020-03-30

\section{Citation:}

Duffy, J. M. N., Hirsch, M., Vercoe, M., Abbott, J., Barker, C., Collura, B., Drake, R., Evers, J. L. H., Hickey, M., Horne, A. W., Hull, M. L., Kolekar, S., Lensen, S., Johnson, N. P., Mahajan, V., Mol, B. W., Otter, A. -S., Puscasiu, L., Rodriguez, M. B. ,... Farquhar, C. M. (2020). A core outcome set for future endometriosis research: an international consensus development study. BJOG-AN INTERNATIONAL JOURNAL OF OBSTETRICS AND GYNAECOLOGY, 127 (8), pp.967-974. https://doi.org/10.1111/1471-0528.16157.

Persistent Link:

http://hdl.handle.net/11343/275573 